\title{
Sonographic Measurement of the Optic Nerve Sheath Diameter (ONSD) and Eyeball Transversal Diameter (ETD) in Benin Healthy African Adults
}

\author{
Kofi-Mensa Savi de Tové $1^{*}$, Djivèdé Akanni' ${ }^{1}$, Miralda Kiki ${ }^{1}$, Sonia Adjadohoun ${ }^{2}$, \\ Godfrid Lokossou', Patricia Yèkpè-Ahouansou' ${ }^{2}$, Olivier Biaou ${ }^{2}$, Vicentia Boco ${ }^{2}$ \\ ${ }^{1}$ Medical Imaging Department, Faculty of Medicine, University of Parakou, Parakou, Republic of Benin \\ ${ }^{2}$ Medical Imaging Department, Faculty of Health Sciences, University of Abomey Calavi, Cotonou, Republic of Benin \\ Email: *savitoveto@yahoo.fr
}

How to cite this paper: de Tové, K.-M.S., Akanni, D., Kiki, M., Adjadohoun, S., Lokossou, G., Yèkpè-Ahouansou, P., Biaou, O. and Boco, V. (2020) Sonographic Measurement of the Optic Nerve Sheath Diameter (ONSD) and Eyeball Transversal Diameter (ETD) in Benin Healthy African Adults. Open Journal of Radiology, 10, 149-158. https://doi.org/10.4236/ojrad.2020.103015

Received: August 31, 2020

Accepted: September 18, 2020

Published: September 21, 2020

Copyright $\odot 2020$ by author(s) and Scientific Research Publishing Inc. This work is licensed under the Creative Commons Attribution International License (CC BY 4.0).

http://creativecommons.org/licenses/by/4.0/

\begin{abstract}
Background: Measuring optic nerve sheath diameter (ONSD), a relatively recent technique, allows an indirect and non-invasive diagnosis of intracranial hypertension. The ONSD ratio to eyeball transverse diameter (ETD) increases this reliability of the technique. The objective of this study was to determine the normal ONSD and its ratio with ETD in black African adults in Benin. Methods: A descriptive cross-sectional study was conducted between May 2019 and August 2019. Ultrasound ONSD and ONSD/ETD ratio were measured in 210 healthy adults received in the medical imaging department of the Borgou and Alibori University Hospital Center in Benin. The ONSD corresponded to the average of 12 measurements ( 03 horizontal and 03 vertical for each eye) taken $3 \mathrm{~mm}$ behind the papilla. The transverse ETD corresponded to the average of 03 measurements. Results: The mean ONSD was $4.20 \pm 0.28 \mathrm{~mm}(95 \% \mathrm{CI}=[4.17 ; 4.24] \mathrm{mm}$ and 95th percentile $=4.70 \mathrm{~mm})$. The mean ETD was $24.25 \pm 1.10 \mathrm{~mm}$. The ONSD/ETD ratio was $0.17 \pm 0.01$ $(95 \% \mathrm{CI}=[0.17 ; 0.17]$ and 95th percentile $=0.19)$. There was no difference between vertical and horizontal ONSD and between right and left ONSD. The ONSD and the ONSD/ETD ratio were not correlated with any of the socio-demographic and anthropometric characteristics studied (sex, age, ethnicity, weight, height, body mass index, head circumference and waist circumference). The ONSD was associated with ETD $(\mathrm{p}<0.001$ and $\mathrm{r}=0.55)$. Conclusion: An ONSD and an ONSD/ETD ratio respectively greater than $4.70 \mathrm{~mm}$ and 0.19 should suspect intracranial hypertension. The ONSD/ETD ratio may be a better indicator of HICT because of its small variation.
\end{abstract}




\section{Keywords}

Optic Nerve Sheath Diameter, Eye Transversal Diameter, Ultrasound, Intracranial Hypertension, Adult, Benin

\section{Introduction}

Intracranial hypertension ( $\mathrm{ICH}$ ) is one of the major causes of mortality and neurological sequelae in neuro-intensive care [1]. Its reference diagnosis method is the intracranial pressure (ICP) monitoring with intra-ventricular or intra-parenchymal sensors [2]. This invasive technique, with possible infectious and/or bleeding complications, is often not available in low and middle incomes countries like Benin. Neuroimaging techniques such as computed tomography (CT), magnetic resonance imaging (MRI) and transcranial Doppler ultrasound allow a non-invasive diagnosis [2] [3] [4]. However, each of them has its limits [2] [5] [6]. As diagnosis alternative, optic nerve sheath diameter (ONSD) measuring, a relatively recent technique was developed [7] [8] [9]. Optic nerve sheath continuity with intracranial subarachnoid spaces explains the increase of its diameter in intracranial hypertension [10]. Sonographic ONSD measuring is a non-invasive, reproductible and easy-to-learn technique, with low intra- and inter-observer variability [11] [12] [13]. In addition, recent studies noted that the association of eye transversal diameter (ETD) measuring through the ONSD/ETD ratio improves the technique's reliability in intracranial hypertension diagnosis [14] [15] [16]. Several studies suggest a DENO variation according to ethnic origin [17] [18] [19] [20]. The efficient use of this technique in the benefit of our populations in Benin, where ultrasound is one of the most accessible imaging techniques, therefore, requires the prior sonographic determination of normal ONSD and ONSD/ETD ratio values, which is the objective of the present study.

\section{Methods}

\subsection{Ethical Approval}

This study was approved by University of Parakou local biomedical research ethics committee and verbal informed consent (in the aboriginal language if necessary) from all subjects included in the study was obtained.

\subsection{Study Design}

This study was performed in the Borgou and Alibori University Hospital medical imaging department at Parakou in Republic of Benin. This cross-sectional descriptive and analytical study with prospective data collection was conducted between May 2019 and August 2019. The sample consisted of subjects recruited by a convenience sampling from patients received in the medical imaging department during the study period and who were at least 19 years of age. 
Subjects with a history of head or orbital-ocular trauma, orbital-ocular pathology that could lead to optic nerve (ON) damage, and those with a pathological abdominal volume increasing were not included. All subjects were examined by a neurologist to rule out any neurological disorders, particularly those suggestive of intracranial hypertension. An ophthalmologist through a clinical examination and a fundus examination has searched and eliminated ocular and optic nerve pathologies.

\subsection{Ultrasound Measurement}

A radiologist with 15-year experience performed the B-mode ultrasound examinations of the optic nerve with a Mindray ultrasound scanner model Digi Prince 8800 plus equipped with $5-10 \mathrm{MHz}$ linear probe. Subjects were examined supine. After applying a thin layer of gel on the closed upper eyelids, the patient was asked to hold the eyeballs immobile in primary visual gaze position. After the optic nerve was correctly individualized, two ultrasonographic sections were carried out: a cross section with the probe positioned horizontally and a sagittal section with the probe positioned vertically. In the hyperechoicretrobulbar fat, the optic nerve sheaths appear hyperechoic surrounding the hypoechoic optic nerve. Measurements were taken $3 \mathrm{~mm}$ behind the papilla and perpendicularly to the optic nerve axis, with the electronics cursors positioned on the sheaths external limits (Figure 1(b)).

To determine a subject's ONSD, six measurements per eye and 12 measurements per subject were performed. An eye's ONSD corresponds to the mean of vertical ONSD measured three times and also the ones of horizontal measured three times. A subject's ONSD equal to the mean of his two eyes ONSD.

The ETD was measured on a cross-section and corresponds to the maximum diameter (retina to retina) obtained by scanning eyeball from upper to lower part (Figure 1(a)). It was measured three times for each eye. An eye's ETD is the mean of the three measurements and a subject ETD is the mean of both eyes ( 6 measurements).

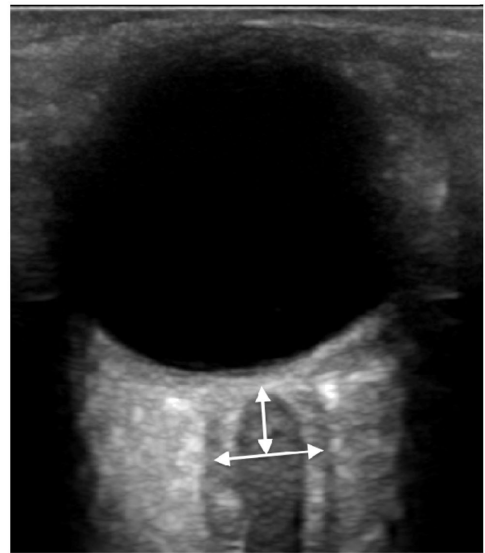

(a)

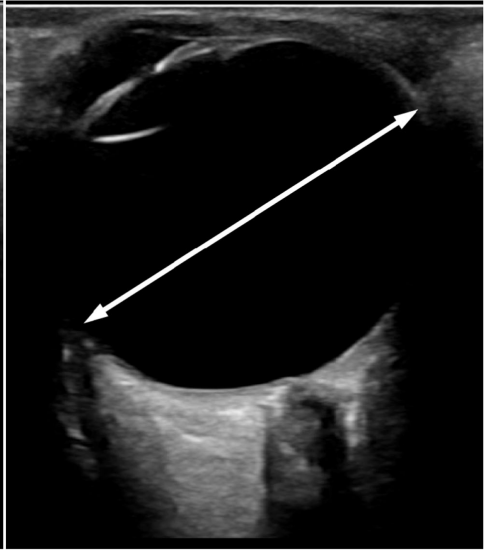

(b)

Figure 1. Ultrasound images showing ETD (a) and of ONSD (b) measurement. 


\subsection{Statistical Analysis}

Variables studied were socio-demographic (age, sex, ethnicity), anthropometric (weight, height, body mass index, head circumference, waist circumference) and sonographic (ONSD, ETD and ONSD/ETD ratio).

Data were analyzed using SPSS for Windows software Release 17.0 (SPSS, Chicago, IL, USA). Correlations were performed using Bravais Pearson's coefficient " $r$ ". A two-tailed probability of $\mathrm{p}<0.05$ was used as significance level.

\section{Results}

\subsection{Study Population Description}

A total of 210 healthy volunteers free of any signs suggestive of intracranial hypertension were enrolled. The mean age was $32 \pm 11.06$ years (range: $19-72$ y). They were $116(55.2 \%)$ males and $94(44.76 \%)$ females. The sex ratio (M/F) was 1.23 .

The anthropometric data mean value was as follow:

- Weight: $66.74 \pm 11.16 \mathrm{~kg}$ (range $38-118 \mathrm{~kg}$ ).

- Height: $1.68 \pm 0.10 \mathrm{~m}$ (range $1.48-1.92 \mathrm{~m}$ ).

- BMI: $23.64 \pm 4.33 \mathrm{~kg} / \mathrm{m}^{2}$ (range $14.65-44.62 \mathrm{~kg} / \mathrm{m}^{2}$ ).

- Head circumference: $55.50 \pm 1.90 \mathrm{~cm}$ (range $45-60 \mathrm{~cm}$ ).

- Waist circumference: $81.80 \pm 11.20 \mathrm{~cm}$ (range $60-118 \mathrm{~cm}$ ).

\subsection{Measurements of ONSD, ETD and ONSD/ETD Ratio}

The mean ONSD was $4.20 \pm 0.28 \mathrm{~mm}(95 \% \mathrm{CI}=[4.17 ; 4.24] \mathrm{mm}$ and 95 th percentile $=4.70 \mathrm{~mm}$ ). The mean ETD was $24.25 \pm 1.1 \mathrm{~mm}$ with an IC95\% $=[24.10$; $24.40] \mathrm{mm}$. The mean ONSD/ETD ratio was $0.17 \pm 0.01$ (95th percentile $=0.19$ ).

Table 1 shows ONSD and ONSD/ETD ratio statistical description of the study population.

ONSD do not vary according to axial or transverse sonographic section plan $(\mathrm{r}=0.98$ and $\mathrm{p}$-value $<0.001)$, nor according to laterality $(\mathrm{r}=0.89$ and $\mathrm{p}$-value $<$ $0.001)$.

In bivariate analysis, head circumference $(\mathrm{r}=0.02$ and $\mathrm{p}$-value $=0.0277)$ and ETD $(r=0.26$ and $p$-value $<0.001)$ were associated with ONSD. In multivariate analysis, only ETD was associated with the ONSD $(r=0.55$ and $\mathrm{p}$-value $<0.001$ (Figure 2).

There was no correlation between ONSD/ETD ratio and socio demographic (age, sex and ethnic group) and anthropometric (weight, height, BMI, head circumference and waist circumference) characteristics of the study population (Table 2).

\section{Discussion}

This study allowed us to determine the mean value of ultrasound ONSD and ONSD/ETD ratio in a healthy black African adult population in Benin. 


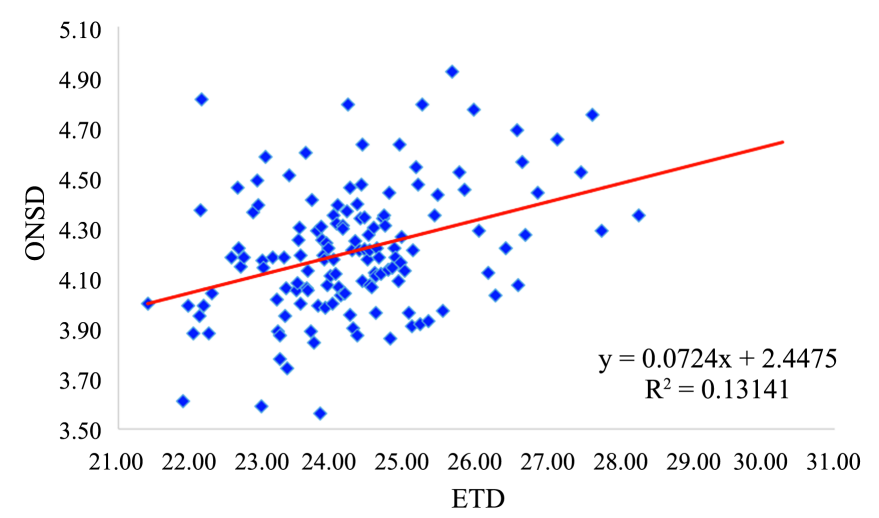

Figure 2. Correlation between ONSD and ETD.

Table 1. Basic descriptive statistics for ONSD, ETD, and the ONSD/ETD ratio.

\begin{tabular}{ccccc}
\hline & Mean $\pm \mathrm{SD}^{\mathrm{d}}\left(\mathbf{9 5 \%} \mathrm{CI}^{\mathrm{e}}\right)$ & Median $\left(\mathrm{IQR}^{\mathrm{f}}\right)$ & Minimum & Maximum \\
\hline ONSD $^{\mathrm{a}}(\mathrm{mm})$ & & & & \\
Vertical & $4.20 \pm 0.28(4.19-4.26)$ & $4.18(4.03-4.43)$ & 3.46 & 5.01 \\
Horizontal & $4.19 \pm 0.28(4.15-4.22)$ & $4.14(3.96-4.36)$ & 3.53 & 5.19 \\
Right & $4.14 \pm 0.28(4.11-4.18)$ & $4.14(3.95-4.33)$ & 3.38 & 4.95 \\
Left & $4.27 \pm 0.30(4.23-4.31)$ & $4.19(4.06-4.44)$ & 3.64 & 5.23 \\
Overall & $4.20 \pm 0.28(4.17-4.24)$ & $4.16(4.00-4.38)$ & 3.54 & 5.07 \\
ETD $(\mathrm{mm})$ & & & & \\
Right & $24.20 \pm 1.10(24.07-24.37)$ & $24.30(23.60-24.80)$ & 21.10 & 27.90 \\
Left & $24.30 \pm 1.10(24.13-24.44)$ & $24.30(23.60-24.70)$ & 21.70 & 28.70 \\
Overall & $24.25 \pm 1.10(24.10-24.40)$ & $24.20(23.70-23.70)$ & 21.40 & 28.30 \\
ONSD/ETD & & $0.17(0.16-0.18)$ & 0.14 & 0.22 \\
Right & $0.17 \pm 0.01(0.17-0.17)$ & $0.17(0.17-0.18)$ & 0.15 & 0.21 \\
Left & $0.18 \pm 0.01(0.18-0.18)$ & $0.17(0.16-0.18)$ & 0.15 & 0.21 \\
Overall & $0.17 \pm 0.01(0.17-0.17)$ & 0.15 & \\
\hline
\end{tabular}

a. optic nerve sheath diameter; b. eyeball transverse diameter; c. ONSD/ETD ratio, d. standard deviation; e. confidence interval; f. interquartile range.

Several studies have determined the normal value of ultrasound-based ONSD in healthy subjects (Table 3). The mean ultrasound ONSD in this study was 4.20 $\pm 0.28 \mathrm{~mm}$, value similar to the one obtained by Blehar et al. [21], Kolade-yunusa et al. [20], Kim et al. [22] and Shrestha et al. [19]. Several authors however have found different values. These include Lochner et al. [19], Bauërle et al. [12], Chen et al. [23] and Lefferts et al. [24] who found greater values and Goeres et al. [25], Wang et al. [26] et Ballantyne et al. [11] who in contrast found smaller values. Less precise linear probes use, sample size and number of measurements performed could explain the observed differences. Factors such as ethnic group difference and variations in "optic nerve" fibremyelination degree could also be responsible for the ONSD size variations observed. 
Table 2. Findings of simple and multiple linear regression for factors associated with ONSD and with ONSD/ETD ratio.

\begin{tabular}{cccc}
\hline & \multicolumn{3}{c}{ p-value } \\
\cline { 2 - 4 } & \multicolumn{2}{c}{ Simple linear regression } & Multiple linear regression \\
\cline { 2 - 4 } & ONSD $^{\mathrm{e}}$ & ONSD/ETD $^{\mathrm{f}}$ & ONSD \\
\hline Age & 0.540 & 0.644 & - \\
Sex & 0.103 & 0.403 & - \\
Ethnic group & 0.084 & 0.144 & - \\
Weight & 0.8447 & 0.49 & - \\
Size & 0.7525 & 0.4486 & - \\
BMI & 0.719 & 0.678 & 0.15 \\
HC $^{\mathrm{b}}$ & $0.0277(\mathrm{r}=0.02)$ & 0.115 & - \\
WC $^{\mathrm{c}}$ & 0.7438 & 0.845 & $<0.001(\mathrm{r}=0.55)$ \\
ETD $^{\mathrm{d}}$ & $<0.001(\mathrm{r}=0.26)$ & - & - \\
\hline
\end{tabular}

a. body mass index; b. headcircumference; c. weast circumference; d. eye transversal diameter; e. optic nerve sheath diameter; f. ONSD/ETD ratio.

Table 3. Summary of published studies estimating ONSD using ultrasonography.

\begin{tabular}{|c|c|c|c|c|c|c|}
\hline First author & Year & Country & $\begin{array}{c}\text { Probe } \\
\text { frequency } \\
(\mathrm{MHz})\end{array}$ & Number $^{\mathrm{a}}$ & Measurements $^{\mathrm{b}}$ & $\begin{array}{r}\text { ONSD }^{c} \\
(\mathrm{~mm})\end{array}$ \\
\hline Lochner [16] & 2016 & Italy & $4-11$ & 20 & 6 & 5.95 \\
\hline Bauërle [12] & 2012 & Germany & $3-9$ & 40 & 6 & 5.4 \\
\hline Chen [23] & 2015 & China & $3-12$ & 519 & 4 & 5.1 \\
\hline Lefferts [24] & 2015 & USA & $7.5-10$ & 20 & 6 & 4.99 \\
\hline Blehar [21] & 2008 & USA & 8.5 & 27 & 4 & 4.3 \\
\hline Kolade-yunusa [20] & 2017 & Nigeria & 7.5 & 150 & 4 & 4.20 \\
\hline Our study & 2019 & Benin & $7.5-10$ & 210 & 12 & 4.20 \\
\hline $\operatorname{Kim}[15]$ & 2017 & South Korea & $3-11$ & 585 & 6 & 4.11 \\
\hline Shrestha [19] & 2016 & Nepal & $6-13$ & 88 & 6 & 4.1 \\
\hline Goeres [25] & 2016 & Canada & $6-13$ & 120 & 4 & 3.68 \\
\hline Wang [26] & 2016 & China & $3-9$ & 230 & 16 & 3.46 \\
\hline Ballantyne [11] & 2002 & Ukraine & 7 & 67 & 6 & $3.2-3.6$ \\
\hline
\end{tabular}

a. Number of healthy subjects; b. Number of mesurements; c. optic nerve sheath diameter.

In our series ONSD, do not vary according to study axis (horizontal or vertical). This finding was also made by Geores and et al. [25] and by Maude and et al. [17]. This can be explained by the optic nerve roughly cylindrical shape [10]. Thus a single sonographic section ONSD's measurement could consequently detect an intracranial hypertension. However, some authors have noted that an ONSD measured on a vertical section was significantly greater than the one 
measured on horizontal section [27].

There was no ONSD variation between the two eyes in our study, as in those of many authors [19] [20] [22] [28] [29]. Therefore, ONSD measurement in the investigation of an intracranial hypertension could be performed on only one eye.

ONSD/ETD ratio measurement is a new approach in the detection of intracranial hypertension. Du et al. in a study of the ONSD/ETD and ultrasound ONSD ratios in the diagnosis of head injury in patients with head injury, found a sensitivity of $90 \%$ and a specificity of $82.3 \%$ for ONSD/ETD versus a sensitivity and specificity of $80 \%$ and $79.3 \%$ respectively for ONSD [16]. In addition to the likely diagnostic accuracy, this ratio has other advantages.

Only ETD was associated with ONSD in our series. Kim et al. [15] [22] and Bekerman et al. [14] had led to the same result. In Wang et al. study, ONSD was associated with gender and BMI [26]. Goeres et al. for their part, found an association only with sex [25].

In this study, the mean ONSD/ETD ratio was $0.17 \pm 0.01$. Kim et al. in their study using ultrasound found a similar value $(0.18 \pm 0.02)$ [22]. A comparable result was obtained by Vaiman et al. who used computed tomography as imaging technique $(0.19 \pm 0.02)$ [30]. Using MRI, Kim et al. found different value $(0.22 \pm 0.01)$ [15]. The better tissue contrast of MRI could explain this difference.

As in many other studies, this survey on, ONSD/ETD ratio was not influenced by socio demographic (age, sex and ethnic group) and anthropometric (weight, height, BMI, head circumference and waist circumference) parameters [15] [16] [30]. The interpretation of ONSD measurement in intracranial hypertension diagnosis should therefore take into account the ETD value. The ratio between these two parameters (ONSD and ETD) shows less inter-individual variation than ONSD.

This study has some limitations. The first is that a direct measurement of intracranial pressure was not performed. This pressure was considered normal based on the clinical information. Furthermore, inter-observer variability could not be studied. This remains a limitation even though several studies have noted low intra- and inter-observer variability [11] [30].

\section{Conclusion}

The mean ONSD in our adult study population was $4.20 \pm 0.28 \mathrm{~mm}$. The ONSD is associated with ETD. The mean DENO/DTG ratio was $0.17 \pm 0.01$. An ONSD and an ONSD/ETD ratio respectively greater than $4.70 \mathrm{~mm}$ and $0.19 \mathrm{~mm}$ in an adult should be considered as increased, suggesting an ICH. This ratio has less inter-individual variability than the ONSD and thus appears to be more suitable for the non-invasive diagnosis of ICH.

\section{Conflicts of Interest}

The authors declare no conflicts of interest regarding the publication of this paper. 


\section{References}

[1] Stevens, R.D., Shoykhet, M. and Cadena, R. (2015) Emergency Neurological Life Support: Intracranial Hypertension and Herniation. Neurocritical Care, 23, 76-82. https://doi.org/10.1007/s12028-015-0168-Z

[2] Raboel, P.H., Bartek, J., Andresen, M., Bellander, B.M. and Romner, B. (2012) Intracranial Pressure Monitoring: Invasive versus Non-Invasive Methods: A Review. Critical Care Research and Practice, 2012, Article ID: 950393. https://doi.org/10.1155/2012/950393

[3] Czosnyka, M., Pickard, J.D. and Steiner, L.A. (2017) Principles of Intracranial Pressure Monitoring and Treatment. Handbook of Clinical Neurology, 140, 67-89. https://doi.org/10.1016/B978-0-444-63600-3.00005-2

[4] Canac, N., Jalaleddini, K., Thorpe, S.G., Thibeault, C.M. and Hamilton, R.B. (2020) Review: Pathophysiology of Intracranial Hypertension and Noninvasive Intracranial Pressure Monitoring. Fluids Barriers CNS, 17, 1-21. https://doi.org/10.1186/s12987-020-00201-8

[5] Krakauskaite, S., Petkus, V., LBartusis, A., Zakelis, R., Chomskis, R., Preiksaitis, A., et al. (2016) Accuracy, Precision, Sensitivity, and Specificity of Noninvasive ICP Absolute Value Measurements. Acta Neurochirurgica (Wien), 122, 317-321. https://doi.org/10.1007/978-3-319-22533-3 63

[6] Evensen, K.B. and Eide, P.K. (2020) Measuring Intracranial Pressure by Invasive, Less Invasive or Non-Invasive Means: Limitations and Avenues for Improvement. Fluids Barriers CNS, 17, 1-33. https://doi.org/10.1186/s12987-020-00195-3

[7] Hansen, H.C. and Helmke, K. (1997) Validation of the Optic Nerve Sheath Response to Changing Cerebrospinal Fluid Pressure: Ultrasound Findings during Intrathecal Infusion Tests. Journal of Neurosurgery, 87, 34-40.

https://doi.org/10.3171/jns.1997.87.1.0034

[8] Hansen, H.C., Lagrèze, W., Krueger, O. and Helmke, K. (2011) Dependence of the Optic Nerve Sheath Diameter on Acutely Applied Subarachnoidal Pressure-An Experimental Ultrasound Study. Acta Ophthalmologica, 89, 528-532. https://doi.org/10.1111/j.1755-3768.2011.02159.x

[9] Liu, D., Li, Z., Zhang, X., Zhao, L., Jia, J., Sun, F., et al. (2017) Assessment of Intracranial Pressure with Ultrasonographic Retrobulbar Optic Nerve Sheath Diameter Measurement. BMC Neurology, 17, 188. https://doi.org/10.1186/s12883-017-0964-5

[10] De Moraes, C.G. (2013) Anatomy of the Visual Pathways. Journal of Glaucoma, 22, S2-S7. https://doi.org/10.1097/IJG.0b013e3182934978

[11] Ballantyne, S.A., O’Neill, G., Hamilton, R. and Hollman, A.S. (2002) Observer Variation in the Sonographic Measurement of Optic Nerve Sheath Diameter in Normal Adults. European Journal of Ultrasound, 15, 145-149. https://doi.org/10.1016/S0929-8266(02)00036-8

[12] Bäuerle, J. and Nedelmann, M. (2012) B-Mode Sonography of the Optic Nerve in Neurological Disorders with Altered Intracranial Pressure. Perspectives in Medicine, 1, 404-407. https://doi.org/10.1016/j.permed.2012.01.001

[13] Lochner, P., Coppo, L., Cantello, R., Nardone, R., Naldi, A., Leone, M.A., et al. (2016) Intra- and Interobserver Reliability of Transorbitalsonographic Assessment of the Optic Nerve Sheath Diameter and Optic Nerve Diameter in Healthy Adults. Journal of Ultrasound, 19, 41-45. https://doi.org/10.1007/s40477-014-0144-Z

[14] Bekerman, I., Sigal, T., Kimiagar, I., Ben Ely, A. and Vaiman, M. (2016) The Quantitative Evaluation of Intracranial Pressure by Optic Nerve Sheath Diameter/Eye Diame- 
ter CT Measurement. American Journal of Emergency Medicine, 34, 2336-2342. https://doi.org/10.1016/j.ajem.2016.08.045

[15] Kim, D.H., Jun, J.-S. and Kim, R. (2018) Measurement of the Optic Nerve Sheath Diameter with Magnetic Resonance Imaging and Its Association with Eyeball Diameter in Healthy Adults. Journal of Clinical Neurology, 14, 345-350. https://doi.org/10.3988/jcn.2018.14.3.345

[16] Du, J., Deng, Y., Li, H., Qiao, S., Yu, M., Xu, Q., et al. (2020) Ratio of Optic Nerve Sheath Diameter to Eyeball Transverse Diameter by Ultrasound Can Predict Intracranial Hypertension in Traumatic Brain Injury Patients: A Prospective Study. Neurocritical Care, 32, 478-485. https://doi.org/10.1007/s12028-019-00762-Z

[17] Maude, R.R., Amir Hossain, M., Hassan, M.U., Osbourne, S., Sayeed, K.L.A., Karim, M.R., et al. (2013) Transorbitalsonographic Evaluation of Normal Optic Nerve Sheath Diameter in Healthy Volunteers in Bangladesh. PLOS ONE, 8, e81013. https://doi.org/10.1371/journal.pone.0081013

[18] Chan, P.Y.N. and Mok, K.L. (2008) Transorbitalsonographic Evaluation of Optic Nerve Sheath Diameter in Normal Hong Kong Chinese Adults. Hong Kong Journal of Emergency Medicine, 15, 197-204. https://doi.org/10.1177/102490790801500402

[19] Shrestha, G.S. (2016) Transorbitalsonographic Evaluation of Normal Optic Nerve Sheath Diameter in Healthy Nepalese Adults. Journal of Neuroanesthesiology and Critical Care, 3, 115-118. https://doi.org/10.4103/2348-0548.182343

[20] Hadijat Oluseyi, K.-Y. and Ukamaka, I. (2017) Ultrasonograhic Measurement of Optic Nerve Sheath Diameter in Normal Adults. Annals of International Medical and Dental Research, 3, 30-34. https://doi.org/10.21276/aimdr.2017.3.2.RD9

[21] Blehar, D.J., Gaspari, R.J., Montoya, A. and Calderon, R. (2008) Correlation of Visual Axis and Coronal Axis Measurements of the Optic Nerve Sheath Diameter. Journal of Ultrasound in Medicine, 27, 407-411. https://doi.org/10.7863/jum.2008.27.3.407

[22] Kim, D.H., Jun, J.S. and Kim, R. (2017) Ultrasonographic Measurement of the Optic Nerve Sheath Diameter and Its Association with Eyeball Transverse Diameter in 585 Healthy Volunteers. Scientific Reports, 7, Article No. 15906. https://doi.org/10.1038/s41598-017-16173-Z

[23] Chen, H., Ding, G.S., Zhao, Y.C., Yu, R.G. and Zhou, J.X. (2015) Ultrasound Measurement of Optic Nerve Diameter and Optic Nerve Sheath Diameter in Healthy Chinese Adults. BMC Neurology, 15, 106.

https://doi.org/10.1186/s12883-015-0361-x

[24] Lefferts, W.K., Hughes, W.E. and Heffernan, K.S. (2015) Effect of Acute High-Intensity Resistance Exercise on Optic Nerve Sheath Diameter and Ophthalmic Artery Blood Flow Pulsatility. Journal of Human Hypertension, 29, 744-748. https://doi.org/10.1038/jhh.2015.12

[25] Goeres, P., Zeiler, F.A., Unger, B., Karakitsos, D. and Gillman, L.M. (2015) Ultrasound Assessment of Optic Nerve Sheath Diameter in Healthy Volunteers. Journal of Critical Care, 31, 168-171. https://doi.org/10.1016/j.jcrc.2015.10.009

[26] Wang, L., Feng, L., Yao, Y., Deng, F., Wang, Y., Feng, J., et al. (2016) Ultrasonographic Evaluation of Optic Nerve Sheath Diameter among Healthy Chinese Adults. Ultrasound in Medicine and Biology, 42, 683-688. https://doi.org/10.1016/j.ultrasmedbio.2015.11.020

[27] Helmke, K. and Hansen, H.C. (1996) Fundamentals of Transorbitalsonographic Evaluation of Optic Nerve Sheath Expansion under Intracranial Hypertension. I. Experimental Study. Pediatric Radiology, 26, 701-705. 
https://doi.org/10.1007/BF01383383

[28] Anas, I. (2014) Transorbitalsonographic Measurement of Normal Optic Sheath Nerve Diameter in Nigerian Adult Population. Malaysian Journal of Medical Sciences, 21, 24-29.

[29] Amini, R., Stolz, L.A., Patanwala, A.E. and Adhikari, S. (2015) Coronal Axis Measurement of the Optic Nerve Sheath Diameter Using a Linear Transducer. Journal of Ultrasound in Medicine, 34, 1607-1612. https://doi.org/10.7863/ultra.15.14.09039

[30] Vaiman, M., Gottlieb, P. and Bekerman, I. (2014) Quantitative Relations between the Eyeball, the Optic Nerve, and the Optic Canal Important for Intracranial Pressure Monitoring. Head \& Face Medicine, 10, Article No. 32.

https://doi.org/10.1186/1746-160X-10-32 